\title{
G-jitter fully developed heat transfer by mixed convection flow in a vertical channel with constant heat flux
}

\author{
Wan Nor Zaleha Amin a , Ahmad Qushairi Mohammad ${ }^{a}$, Mohammed Abdulhameed ${ }^{b}$, Sharidan \\ Shafie ${ }^{a,{ }^{*}}$
}

a Department of Mathematical Sciences, Faculty of Science, Universiti Teknologi Malaysia, 81310 UTM Johor Bahru, Johor, Malaysia

b School of Science and Technology, Federal Polytechnic, P.M.B., Bauchi 0231, Nigeria

* Corresponding author: sharidan@utm.my

\section{Article history}

Received 9 January 2019

Revised 19 March 2019

Accepted 2 May 2019

Published Online 2 February 2020

\section{Graphical abstract}

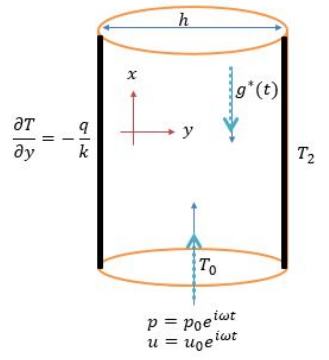

\begin{abstract}
A theoretical study of mixed convection heat transfer was carried out in an infinite length of vertical channel with both open ends. One of the vertical plates was prescribed with constant heat flux. The effect of $g$-jitter was also taken into consideration. The Fourier method was utilized to solve the resulting governing equations. The behavior of the fluid temperature and velocity of the flow were studied and presented graphically in this paper. The graphical results were later on analyzed and discussed. The behavior of steady state flow was also investigated. Results confirmed that as wall temperature increased, the fluid temperature increased. The velocity increased due to increments in mixed convection and oscillation parameter, on the other hand, it decreased as a frequency of $\mathrm{g}$ jitter increased.
\end{abstract}

Keywords: Mixed convection, vertical channel, heat transfer, constant heat flux, g-jitter

\section{INTRODUCTION}

The fundamental study of heat transfer is crucial in many industrial sectors. Hence, the analysis of the theoretical and experimental study is sufficiently great and acknowledged to be worthy of attention. There is a broad study of the heat transfer that can be implemented in the industrial sector such as a production of metal and cooling of the electronic equipment. Due to this reason, there are numerous studies have been carried out by past researchers on the behavior of the fluid in certain geometry of a system over the past years.

Therefore, a paper by Sajid et al. (2010) dealt with fully developed mixed convection flow of viscoelastic fluid in vertical plates. An exact solution was implemented into the equations to seek the results of temperature and velocity distributions. The results showed that as the viscoelastic and cross-flow parameter increased, the velocity will be decreased. However, as Prandtl and Grashof numbers increased, the velocity increased whereas temperature decreased when Reynolds number increased.

Furthermore, Sivasamy et al. (2010) conducted a research on mixed convection flow of a porous channel, numerically employed Darcy model and found that the increasing values of Grashof and Reynolds numbers will increase the Nusselt numbers.

Later, Roşca and Pop (2013) studied the mixed stagnation point past vertical plate with second order slip using bvp $4 \mathrm{c}$ from MATLAB with constant heat flux. The paper performed stability analysis to verify stability of the results and established that the second order slip affected considerably the heat and flow characteristics.

Recently, Altunkaya et al. (2017) conducted a research to investigate analytically an open-ended vertical microchannel using perturbation technique with constant heat flux. The author performed the analysis on Nusselt numbers, $\mathrm{Nu}$ against the mixed convection parameter, rarefaction, and viscous dissipation. The results exemplified that as mixed convection and rarefaction increased, the value of $\mathrm{Nu}$ will decrease.

Moreover, there are many numerical studies on mixed convection flow with the effect of g-jitter. One of them was a research by Shafie and Amin (2005) that studied free convection of a sphere, subjected to constant heat flux using implicit finite-difference scheme. In addition, Saeid (2006) also applied the same method to solve the problem of gjitter-induced free convection flow over a vertical flat plate. It was followed by Naser et al. (2013) who conducted a numerical study of gjitter on stretching sheet.

The presented review of literature suggested that little attention has been paid to evaluate mixed convection from discretely heated curved surfaces, and from a foregoing discussion, it is clear that no prior results are available for opposing mixed convection heat transfer in channels with an effect of g-jitter with a prescribed heat flux boundary condition. In particular, this research was proposed to expand the work by Sharidan et al. (2005) which investigated analytically the effect of gjitter on mixed convection flow in two vertical parallel plates.

Furthermore, a case of vertical channel or also known as two vertical parallel plates with an effect of g-jitter has not yet been considered in any fluid flow model. In respect of that, the Fourier method was employed to simplify the fluid flow model of the mixed convection in a vertical channel. One of the main factors that contributed to this research was lack of reference data of the analytical approach for their validation. As a result, these exact solutions can be used to check the correctness for solutions of more complex mathematical models, obtained through numerical schemes. 
In this paper, detailed analytical approach of mixed convection heat transfer in a vertical channel with both open ends of infinitely length with prescribed constant heat flux was carried out to investigate behavior of a fluid flow and thermal. The mathematical models were solved analytically using Fourier method, subjected to accurate initial and boundary conditions. Certain values of the considerable parameters were proposed in this research to study a pattern of velocity and temperature profiles.

In many applications, constant heat flux is likely being isothermal especially in electric components in the circuit board. Thus, it is important to understand effects of g-jitter in designing and maintaining the cooling board of a system. Considering this impact, the present research was established to prevent such overheating problems such as explosion in practical application. In this context, the present work aimed at contributing to future understanding of theoretical knowledge of fluid flow and thermal performance of such effects to behavior of fluid motion.

\section{MATHEMATICAL MODEL}

This research considered an incompressible fluid which unsteadily flowed between two vertical parallel plates with width, $h$ and uniform temperatures at the walls. A stationary Cartesian coordinate was chosen such that the $x$-coordinate extended along the surface and $y$-coordinate was normal to it. Pressure gradient of oscillatory type was applied in the flow direction. The presence of fully developed g-jitter effect was also considered. Assuming at time, $t=0$, the flow is at a constant temperature, $T_{0}$ and has mean velocity, $u_{0}$ and pressure, $p_{0}$. The assumption of fully developed flow means that the axial ( $x$-direction) velocity solely depends on the transverse coordinate, $y$. In that case, from continuity equation, the transverse velocity, $v$ must be zero. The axial velocity, $u$ and the fluid temperature, $T$ are assumed to be functions of $y$ plus function of time, $t$. The pressure variation is found to be a linear function of $x$ and $t$, and assumed that the gravity acceleration is given by $g^{*}(t)=g_{0} \sin (\omega t)$ in which $g_{0}$ is magnitude and $\omega$ is frequency of g-jitter field.

In the view of these assumptions, the initial and boundary layer conditions governing a mixed convection flow of fluid passed an oscillating vertical plate containing continuity equation, an equation of motion and energy equation can be casted into the following forms:

$$
\begin{gathered}
\nabla \cdot \boldsymbol{u}=\frac{\partial u}{\partial x}+\frac{\partial v}{\partial y}=0 \\
\rho \frac{\partial u}{\partial t}=-\frac{\partial p}{\partial x}+\mu \frac{\partial^{2} u}{\partial y^{2}}+g^{*}(t)\left(\rho \beta_{T}\right)\left(T-T_{0}\right), \\
\frac{\partial T}{\partial t}=\frac{k}{\left(\rho c_{p}\right)} \frac{\partial^{2} T}{\partial y^{2}} .
\end{gathered}
$$

which subjected to

$$
\begin{aligned}
& t<0 \text { : } \\
& u=0, T=T_{0} \\
& t \geq 0 \text { : } \\
& \begin{array}{c}
u=0, \frac{\partial T}{\partial y}=-\frac{q}{k} \\
u=0, T=T_{2}
\end{array} \\
& \text { for } 0<y<h \\
& \text { at } y=0 \\
& \text { at } \quad y=h
\end{aligned}
$$

in which $\mu$ is dynamic viscosity of fluid, $\rho$ is fluid density, $\beta_{T}$ is volumetric coefficient of thermal expansion of fluid, $v$ is kinematic viscosity, $p$ is fluid pressure, $k$ is thermal conductivity of fluid, $c_{p}$ is specific heat of fluid of a constant pressure, $q$ is heat flux, $h$ is a width of channel, and $T_{2}$ is temperature of the right wall.

Next, Equations (2) and (3) are transformed into the following nondimensional equations

$$
\begin{gathered}
\frac{\partial U}{\partial \tau}=-\frac{\partial P}{\partial X}+\frac{\partial^{2} U}{\partial Y^{2}}+\frac{G r}{R e} \cdot g(\tau) \cdot \theta, \\
\frac{\partial \theta}{\partial \tau}=\frac{1}{\operatorname{Pr}} \frac{\partial^{2} \theta}{\partial Y^{2}}
\end{gathered}
$$

in which non-dimensional variables are defined as

$$
\begin{gathered}
U=\frac{u}{u_{0}}, \tau=\frac{v t}{h^{2}}, P=\frac{h p}{\rho v u_{0}}, X=\frac{x}{h}, Y=\frac{y}{h} \\
\theta=\frac{T-T_{0}}{h q / k}, g(\tau)=\frac{g^{*}}{g_{0}}
\end{gathered}
$$

with Grashof number, $G r$, Reynolds number, $R e$, and Prandtl number, $\mathrm{Pr}$ are given by

$$
G r=\frac{g_{0} \beta_{T} q h^{4}}{k v^{2}}, R e=\frac{u_{0} h}{v}, \operatorname{Pr}=\frac{v \rho c_{p}}{k} .
$$

Then, the initial and boundary conditions (4) become

$$
\begin{array}{rrrrrl}
\tau<0: & U=0, \theta=0 & \text { for } & & 0<Y<1 \\
\tau \geq 0: & U=0, \frac{\partial \theta}{\partial y}=-1 & \text { at } & & Y=0 \\
& & & \text { at } & & Y=1
\end{array}
$$

in which the parameter of non-dimensional wall temperature, $r_{T}$ is defined as

$$
r_{T}=\frac{T_{2}-T_{0}}{h q / k} .
$$

Before solving Equation (5), Equation (6) needs to be solved first. At this time, using separation of variables method by considering $\theta(Y, \tau)=Y(y) \tau(t)$, Equation (6) has a solution of

$$
\theta(Y, \tau)=1+r_{T}-Y .
$$

Subsequently, Equation (5) is solved using the same method as Equation (6). It is assumed that $U=e^{i \Omega \tau} \cdot \Phi(Y), P=e^{i \Omega \tau} \cdot F(X)$ and $g(\tau)=e^{i \Omega \tau}$ [Sharidan et al. (2005)]. Thus, Equation (5) becomes

$$
i \Omega e^{i \Omega \tau} \Phi=-\frac{\partial F}{\partial X} e^{i \Omega \tau}+\Phi " e^{i \Omega \tau}+\frac{G r}{R e} \cdot e^{i \Omega \tau} \cdot\left[1+r_{T}-Y\right]
$$

along with the boundary conditions

$$
\Phi(0)=0, \Phi(1)=0 .
$$

Further, condition of conservation of mass is defined as

$$
\int_{0}^{1} \Phi(Y) d Y=1
$$

Hence, a general solution of $\Phi(Y)$ becomes

$$
\begin{aligned}
\Phi(Y)=\frac{1}{\beta^{2}}\left\{\left(\frac{\partial F}{\partial X}-\right.\right. & \left.\frac{G r}{R e}\left[1+r_{T}\right]\right) \frac{\sinh (\beta(1-Y))}{\sinh \beta} \\
& +\left(\frac{\sinh \beta Y}{\sinh \beta}\left\{\frac{\partial F}{\partial X}-\frac{G r}{R e}\left(r_{T}\right)\right\}\right) \\
& \left.+\frac{G r}{R e}\left[1+r_{T}-Y\right]-\frac{\partial F}{\partial X}\right\}
\end{aligned}
$$

in which $\beta^{2}=\mathrm{i} \Omega$ with $\partial F / \partial X$ is given by

$$
\frac{\partial F}{\partial X}=\frac{\beta^{3} \sinh \beta}{2 \cosh \beta-\beta \sinh \beta-2}+\frac{G r}{R e}\left[\frac{1+2 \cdot r_{T}}{2}\right] .
$$

Now, for the case of steady flow where $\Omega=0$, the gravity field is constant. Therefore, the solution is set as

$$
\Phi(Y)=\frac{G r}{R e}\left[\left(\frac{Y}{12}-\frac{Y^{2}}{4}+\frac{Y^{3}}{6}\right)\right]+6\left(Y-Y^{2}\right)
$$


The solutions of temperature (11) and velocity profiles (15) satisfy the boundary condition (9) and Equations (5) and (6). Hence, the solutions are verified.

\section{RESULTS AND DISCUSSION}

The results were then computed into mathematical software named MATLAB and various values of the desired parameters were generated and presented graphically. The quantities plotted along the vertical axis were temperature and velocity profiles that the equations of solutions are as follows:

$$
\begin{gathered}
\theta(Y, \tau)=1+r_{T}-Y, \\
\operatorname{Imag}\left(U=\Phi e^{i \Omega \tau}\right)=\operatorname{Real}(\Phi) \sin (\Omega \tau)+\operatorname{Imag}(\Phi) \cos (\Omega \tau)
\end{gathered}
$$

It represents a value of driving force

$$
g^{*}(t)=\operatorname{Imag}\left(g_{0} e^{i \omega t}\right) \text { and } u_{0}(t)=u_{0} \sin (\omega t) .
$$

Fig. 1 illustrates the effects of non-dimensional wall temperature, $r_{T}$ on temperature distribution. The values of $r_{T}$ were in the range of $0 \leq r_{T} \leq 1$. Form the graph, as $r_{T}$ increases, $\theta$ also increases. This is due to the fact that a value of $r_{T}$ is directly proportional to the temperature, $\theta$. As $r_{T}$ is increasing, more hot fluid is carried through a vertical channel due to the increasing fluid temperature which consequently results in higher wall temperature gradient.
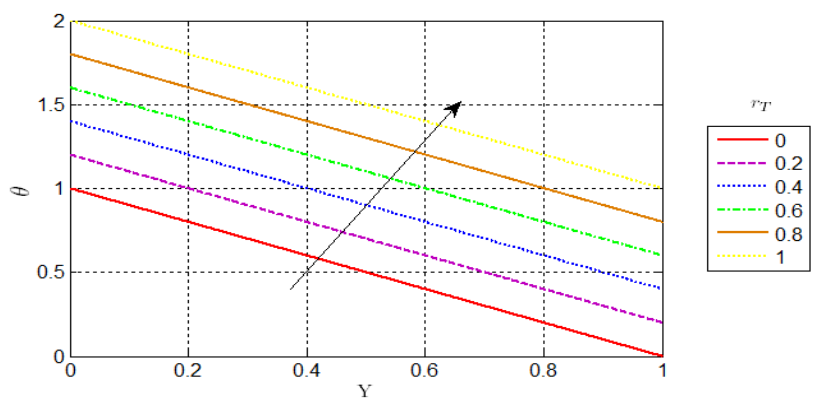

Fig. 1 Temperature profiles for different values of $r_{T}$.

Figs. 2 to 6 illustrate the results for velocity profiles of consistent values of parameters. The parameters incorporated in the problem were oscillating, $\Omega \tau$, frequency, $\Omega$ and mixed convection parameter, $\mathrm{Gr} / \mathrm{Re}$.

Results of analysis on effects of mixed convection parameter, $\mathrm{Gr} / \mathrm{Re}$ on the fluid flow are shown in Fig. 2. It was found that the velocity of the fluid increases when $\mathrm{Gr} / \mathrm{Re}$ increases. Velocity profile is always symmetric about a centerline of the channel that $Y=0.5$.
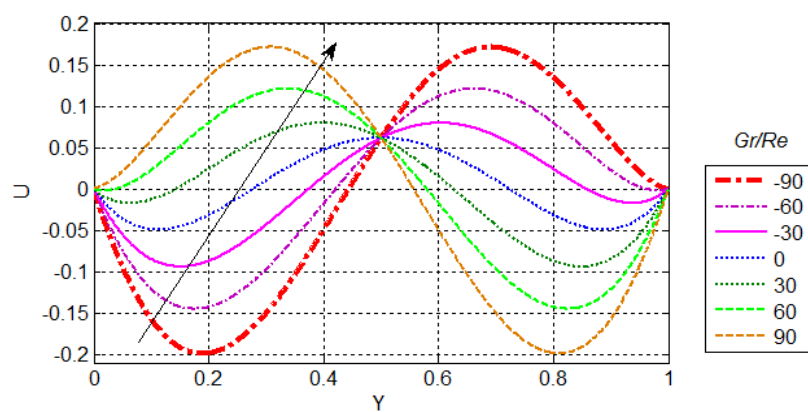

Fig. 2 Velocity profiles for different values of Gr/Re with $\mathrm{r}_{T}=0.3, \Omega \tau=$ $\pi$ and $\Omega=10$.

Fig. 3 represents the effects of oscillating parameter, $\Omega \tau$ towards the behavior of fluid velocity, U. In Figs. 3(a) and (b), it can be observed that there was only a small reversed flow closed to the left wall for $\mathrm{Gr} / \mathrm{Re}=30$ and increasing reversed flow close to the left wall when $\mathrm{Gr} / \mathrm{Re}=300$, respectively. In Fig. 3(a), a velocity profile shows a full reversed flow when oscillating parameter, $\Omega \tau$ is increasing while in Fig. 3 (b), there is a partial reversed flow when $\Omega \tau$ increases. Velocity of fluid fluctuates with increasing values of $\Omega \tau$. It is apparent that fluid flows more frequent as $\mathrm{Gr} / \mathrm{Re}$ increases.
To make a comparison, different values of non-dimensional wall temperature, $r_{T}$ were considered as shown in Fig. 4. For different values of $r_{T}$, velocity profile does not change when $r_{T}$ is increased. The results show that the values of $r_{T}$ does not affect the velocity profile at all.

(a)

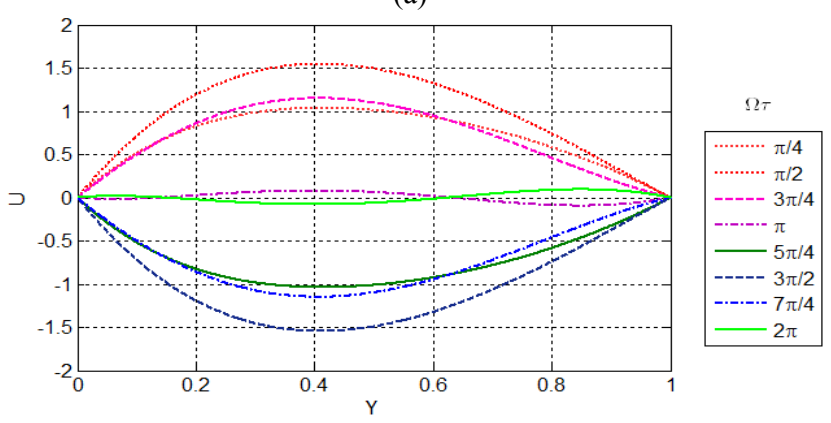

(b)

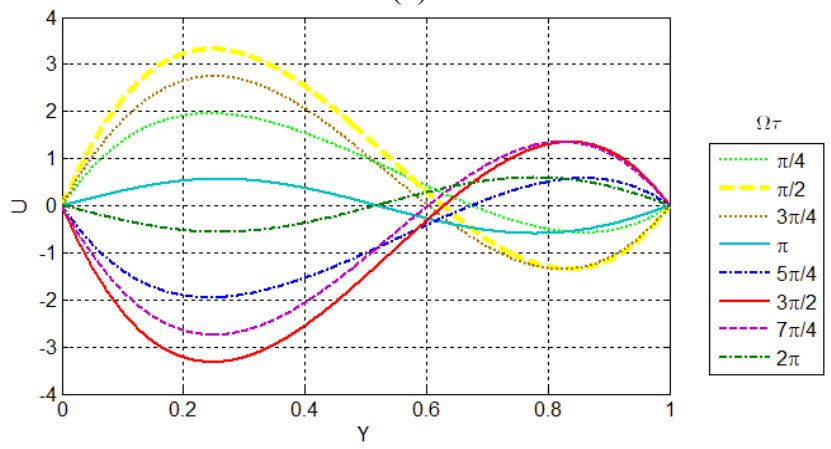

Fig. 3 Velocity profiles for different values of $\Omega \tau$ with $r_{T}=0.3$ and $\Omega=10$; a) $\mathrm{Gr} / \mathrm{Re}=30$; b) $\mathrm{Gr} / \mathrm{Re}=300$.

(a)

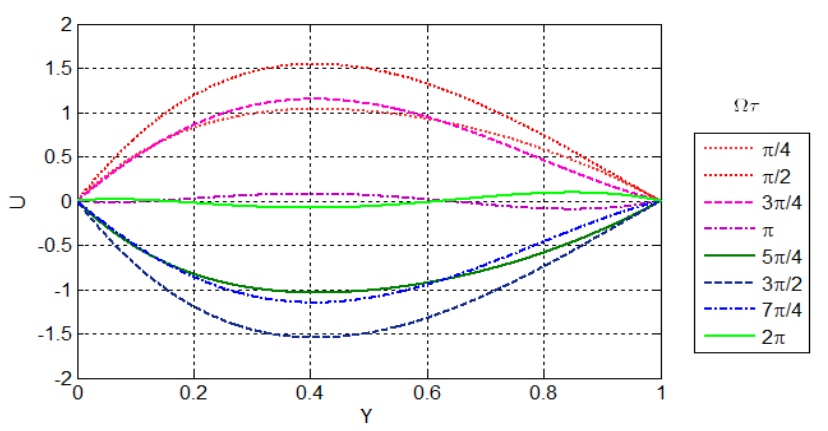

(b)
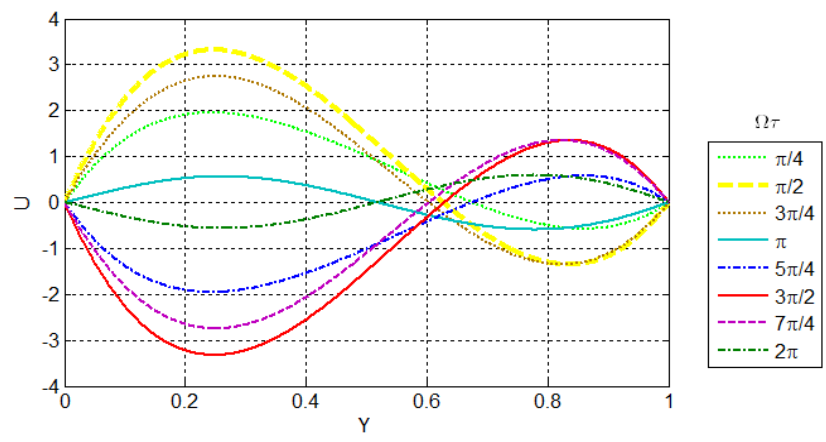

Fig. 4 Velocity profiles for different values of $\Omega \tau$ with $r_{T}=0.7$ and $\Omega=10$ a) $\mathrm{Gr} / \mathrm{Re}=30$; b) $\mathrm{Gr} / \mathrm{Re}=300$.

Furthermore, Fig. 5 illustrates velocity profiles for different values of frequency, $\Omega$. The result verifies that there is merely a small reversed flow close to the right wall for $\frac{\mathrm{Gr}}{\mathrm{Re}}=30$ as shown in Fig. 5(a) but it increases when $\mathrm{Gr} / \mathrm{Re}=300$ as shown in Fig. 5(b). The results agree that as frequency increases, the velocity decreases. Therefore, the frequency of g-jitter is inversely proportional to the velocity. It 
indicates that a fluid flow decelerates with the increment of the frequency. It has been confirmed that as $\Omega$ increases, a velocity fluctuates. Moreover, a fluid flow is less frequent as $\mathrm{Gr} / \mathrm{Re}$ increases.

(a)

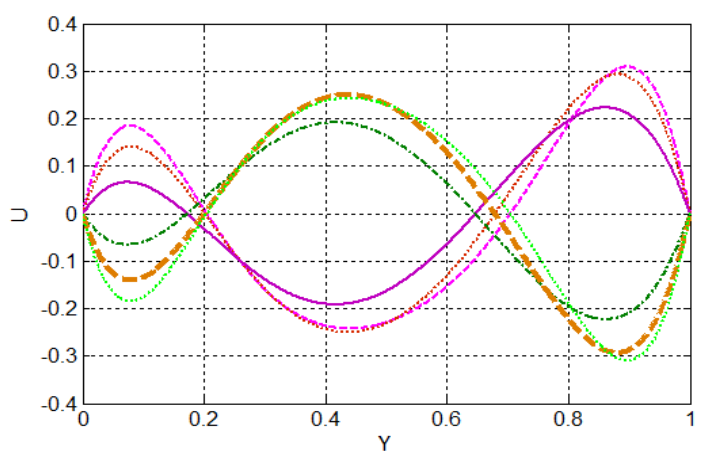

(b)
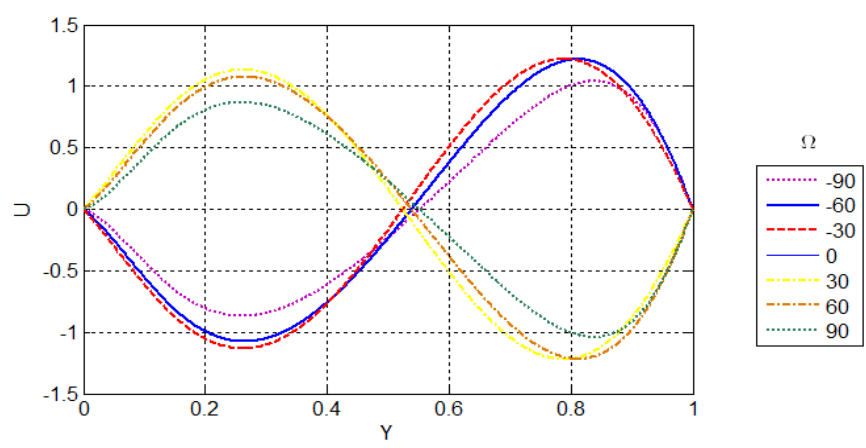

Fig. 5 Velocity profiles for different values of $\Omega$ with $r_{T}=0.3, \Omega \tau=\pi$ (a) $\mathrm{Gr} / \mathrm{Re}=30$; (b) $\mathrm{Gr} / \mathrm{Re}=300$.

Finally, for the case of steady flow in which $\Omega=0$, Equation (17) was computed and presented graphically in Fig. 6. The result proves that the velocity profile is always symmetric about the centerline of a channel which is $Y=0.5$. Therefore, as a mixed convection parameter, as $\mathrm{Gr} / \mathrm{Re}$ increases, the velocity will be increased. The presence of mixed convection has enabled the researchers to seek the consequence of a relaxation time on the fluid velocity.

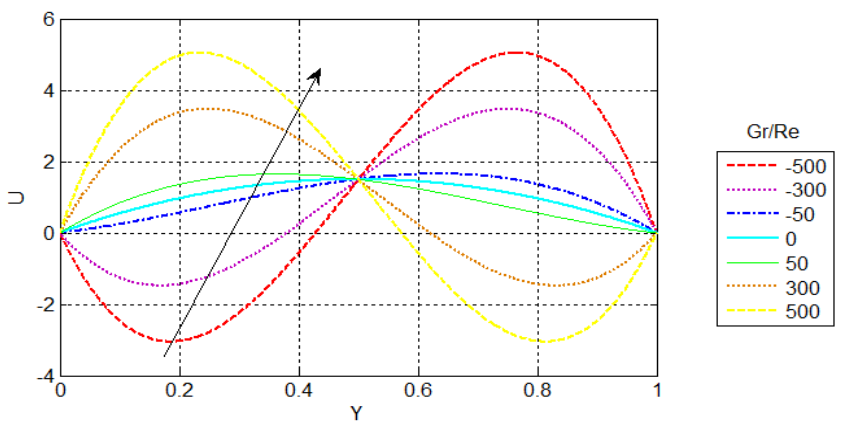

Fig. 6 Velocity profiles steady state for different values of $\mathrm{Gr} / \mathrm{Re}$ with $\Omega=$ 0 .

\section{CONCLUSION}

This paper focused on heat transfer mixed convection flow in a vertical channel with constant heat flux. The mechanism of the heat transfer was modeled in the existence of g-jitter effect. It can be concluded that temperature, $\theta$ was directly proportional to wall temperature, $r_{T}$. Additionally, velocity, $U$ fluctuated due to frequent number of oscillations of fluid parameter, $\Omega \tau$. Meanwhile, the velocity, $U$ increased as $\mathrm{Gr} / \mathrm{Re}$ increased whereas velocity decreased when a frequency of g-jitter increased. The velocity remained unchanged when a non-dimensional wall temperature, $r_{T}$ increased. For the case of steady flow, the results obtained demonstrated that the velocity, $U$ increased when $\mathrm{Gr} / \mathrm{Re}$ increased.

\section{ACKNOWLEDGEMENT}

The authors would like to acknowledge the Ministry of Education (MOE) and Research Management Centre-UTM for their financial support through vote numbers 5F004, 07G70, 07G72, 07G76, and 07G77.

\section{REFERENCES}

Sajid, M., Pop, I. and Hayat, T. 2010. Fully developed mixed convection flow of a viscoelastic fluid between permeable parallel vertical plates. Computers \& Mathematics with Applications, 59(1), 493-498.

Sivasamy, A., Selladurai, V. and Rajesh Kanna, P. 2010. Mixed convection on jet impingement cooling of a constant heat flux horizontal porous layer. International Journal of Thermal Sciences, 49(7), 1238-1246.

Roșca, N. C. and Pop, I. 2013. Mixed convection stagnation point flow past a vertical flat plate with a second order slip: Heat flux case. International Journal of Heat and Mass Transfer, 65, 102-109.

Altunkaya, A. N., Avci, M. and Aydin, O. 2017. Effects of viscous dissipation on mixed convection in a vertical parallel-plate microchannel with asymmetric uniform wall heat fluxes: The slip regime. International Journal of Heat and Mass Transfer, 111, 495-499.

Shafie, S. and Amin, N. 2005. g-Jitter induced free convection boundary layer on heat transfer from a sphere with constant heat flux. Malaysian Journal of Fundamental and Applied Sciences, 1(1).

Saeid, N. H. 2006. G-jitter induced free convection over a vertical flat plate. ASEAN Journal on Science and Technology for Development, 23(1\&2), 133-143.

Naser, N., Kasim, A. R. M. and Aurangzaib, S. S. 2013. g-Jitter mixed convection on double diffusion adjacent to a vertical stretching sheet. Recent Advances in Mathematics, 7, 174-181. Cambridge, MA, USA: WSEAS Press.

Sharidan, S., Amin, N. and Pop, I. 2005. G-jitter fully developed combined heat and mass transfer by mixed convection flow in a vertical channel. International communications in heat and mass transfer, 32(5), 657-665. 